ACCEPTED MANUSCRIPT

\title{
Electron power absorption dynamics in magnetized capacitively coupled radio frequency oxygen discharges
}

To cite this article before publication: Li Wang et al 2020 Plasma Sources Sci. Technol. in press https://doi.org/10.1088/1361-6595/abb2e7

\section{Manuscript version: Accepted Manuscript}

Accepted Manuscript is "the version of the article accepted for publication including all changes made as a result of the peer review process, and which may also include the addition to the article by IOP Publishing of a header, an article ID, a cover sheet and/or an 'Accepted

Manuscript' watermark, but excluding any other editing, typesetting or other changes made by IOP Publishing and/or its licensors"

This Accepted Manuscript is @ 2020 IOP Publishing Ltd.

During the embargo period (the 12 month period from the publication of the Version of Record of this article), the Accepted Manuscript is fully protected by copyright and cannot be reused or reposted elsewhere.

As the Version of Record of this article is going to be / has been published on a subscription basis, this Accepted Manuscript is available for reuse under a CC BY-NC-ND 3.0 licence after the 12 month embargo period.

After the embargo period, everyone is permitted to use copy and redistribute this article for non-commercial purposes only, provided that they adhere to all the terms of the licence https://creativecommons.org/licences/by-nc-nd/3.0

Although reasonable endeavours have been taken to obtain all necessary permissions from third parties to include their copyrighted content within this article, their full citation and copyright line may not be present in this Accepted Manuscript version. Before using any content from this article, please refer to the Version of Record on IOPscience once published for full citation and copyright details, as permissions will likely be required. All third party content is fully copyright protected, unless specifically stated otherwise in the figure caption in the Version of Record.

View the article online for updates and enhancements. 


\section{Electron power absorption dynamics in magnetized capacitively coupled radio frequency oxygen discharges}

Li Wang ${ }^{1,2}$, De-Qi Wen ${ }^{3}$, Peter Hartmann ${ }^{4}$, Zoltán Donkó ${ }^{4}$, Aranka Derzsi ${ }^{4}$, Xi-Feng Wang $^{1,5}$, Yuan-Hong Song ${ }^{1 *}$, You-Nian Wang $^{1}$, and Julian Schulze ${ }^{1,2}$

${ }^{1}$ Key Laboratory of Materials Modification by Laser, Ion, and Electron Beams (Ministry of Education), School of Physics, Dalian University of Technology, Dalian 116024, People's Republic of China

${ }^{2}$ Department of Electrical Engineering and Information Science, Ruhr-University Bochum, D-44780, Bochum, Germany

${ }^{3}$ Department of Computational Mathematics Science and Engineering, Michigan State University, East Lansieg, USA

${ }^{4}$ Institute for Solid State Physics and Optics, Wigner Research Centre for Physics, H-1121 Budapest, Konkoly-Thege Miklós str. 29-33, Hungary

${ }^{5}$ Department of Electrical Engineering and Computer Science, University of Michigan, 1301 Beal Ave., Ann Arbor, MI, 48109-2122, USA

*E-Mail: songyh@dlut.edu.cn 
The influence of a uniform magnetic field parallel to the electrodes on radio frequency capacitively coupled oxygen discharges driven at $13.56 \mathrm{MHz}$ at a pressure of $100 \mathrm{mTorr}$ is investigated by one-dimensional Particle-in-Cell/Monte Carlo collision (1D PIC/MCC) simulations. Increasing the magnetic field from 0 to $200 \mathrm{G}$ is found to result in a drastic enhancement of the electron and the $\mathrm{O}_{2}^{+}$ion density due to the enhanced confinement of electrons by the magnetic field. The time and space averaged $\mathrm{O}^{-}$ion density, however, is found to remain almost constant, since both the dissociative electron attachment (production channel of $\mathrm{O}^{-}$) and the associative electron detachment rate due to the collisions of negative ions with oxygen metastables (main loss channel of $\mathrm{O}^{-}$) are enhanced simultaneously. This is understood based on a detailed analysis of the spatio-temporal electron dynamics. The nearly constant $\mathrm{O}^{-}$density in conjunction with the increased electron density causes a significant reduction of the electronegativity and a pronounced change of the electron power absorption dynamics as a function of the externally applied magnetic field. While at low magnetic fields the discharge is operated in the electronegative Drift-Ambipolar (DA) mode, a transition to the electropositive $\alpha$-mode is induced by increasing the magnetic field. Meanwhile, a strong electric field reversal is generated near each electrode during the local sheath collapse at high magnetic fields, which locally enhances the electron power absorption. A model of the electric field generation reveals that the reversed electric field is caused by the reduction of the electron flux to the electrodes due to their trapping by the magnetic field. The consequent changes of the plasma properties are expected to affect the applications of such discharges in etching, deposition and other semiconductor processes.

Keywords: capacitively coupled oxygen plasmas, magnetized plasma, electric field reversal, electron dynamies




Low temperature radio frequency capacitively coupled plasmas (RF CCPs) are commonly applied in surface etching, deposition and sputtering devices in microelectronic mamufacturing $[1-3]$. For these applications, appropriate and controllable plasma properties such as the plasma density, the Electron Energy Distribution Function (EEDF), theIon Energy Distribution Function (IEDF) at the boundary surfaces, and the plasma uniformity are required for an optimum efficiency and quality of material processing on microscopic scales [4-7]. Magnetized CCPs are frequently used for such applications and have shown good performance in improving distinct plasma properties, especially the plasma density [8-10]. Typically the magnetic field is oriented parallel to the electrodes to limit the cross-field transport of electrons to the walls. In this way the electron losses are/reduced and the electron power absorption as well as the plasma density are enhanced $[11,12]$.

In order to realize separate control of the ion flux and mean ion energy at the electrodes, tailored voltage waveforms (TVW) are often used in unmagnetized CCP discharges [13, 14]. Such waveforms are generated by the superposition of a fundamental frequency and its higher harmonics with adjustable amplitudes and phases. By changing the phase angles between the driving harmonics, the ion energy could be tuned by controlling the DC self bias via the Electrical Asymmetry Effect (EAE). [15, 16]. For such a system, the presence of a magnetic field parallel to the electrodes was found to increase the ion flux to the electrodes significantly and almost independently of the phase between the driving harmonics [17, 18]. In geometrically and electrically symmetric single-frequency discharges, an axially nonuniform magnetic field was demonstrated to induce a Magnetic Asymmetry Effect (MAE). Tuning the magnetic field strength at a reference distance from one of the electrodes was found to allow control of the DC self bias and, thus, the IEDF at the electrodes [19]. Moreover, the ion flux can be adjusted in this way [20]. Oberberg et al. [21] also found that tuning such axially non-uniform magnetic fields in low pressure CCPs allows to control the self-excitation of the plasma series resonance (PSR) and Non-Linear Electron Resonance Heating (NERH) in space and time due to the magnetic control of the plasma symmetry $[22,23]$. Other recent studies reported that a homogeneous B-field can lead to an asymmetry in CCP discharges and can improve the control of the ion energy and flux at boundary surfaces [24]. These previous results indicate that the application of a magnetic field can 
induce significant changes of the electron dynamics and of the plasma characteristics. If these effects can be understood and controlled, they could provide new concepts for knowledge based optimization of materials processing.

Electron power absorption from electromagnetic fields in CCP discharges is a fundamental and important phenomenon. The spatio-temporal electron dynamics largely determine the space and time dependent EEDF and, thus, radical generation and the formation of charged particle distribution functions in the plasma volume and at boundary surfaces. In unmagnetized CCP discharges, these phenomena have been investigated for a variety of discharge conditions [25]. Several electron power absorption modes have been identified, which are mainly determined by the presence of different gases and the choice of external control parameters such as the applied power (or voltage), the driving frequency as well as the neutral gas pressure. For example, in electropositive discharges, the ' $\alpha$-mode' [26] and the ' $\gamma$-mode' [27], where electrons are accelerated by electric fields during the times of sheath expansion within each RF period and the strong electric field inside sheaths, respectively, are the most common electron power absorption modes. However, in electronegative discharges, the depletion of the electron density plays an important role for the electron power absorption [7, 28-30]. In strongly electronegative discharges, the low electron density leads to the presence of strong drift electric fields in the bulk region as well as of strong ambipolar fields near the sheath edges. This is known as the Drift-Ambipolar (DA) mode [31]. Besides the DA mode, a striation (STR) mode was also observed in electronegative discharges operated at conditions when both the positive and negative ions can react to the dynamics of the electric field inside the discharge [32]. Transitions between these power absorption modes have been observed in different gases by changing external control parameters. For instance, in oxygen CCPs, a transition from the DA-mode to the $\alpha$-mode has been found to be induced by changing the gas pressure [33, 34], the driving frequency [35, 36], the driving voltage waveform [7, 28, 36-38], as well as the gap distance [34, 39].

All of the above electron power absorption modes are based on electron motion along the direction perpendicular to the electrodes. However, in the presence of a magnetic field, electrons also perform a gyro-motion around the magnetic field lines. If the magnetic field is parallel to the electrodes, the electrons also experience an $\mathbf{E} \times \mathbf{B}$ drift that is parallel to the electrode surfaces. At such conditions, the mechanisms of electron power absorption are expected to be modified. Turner et al. [40] investigated the electron power absorption in a 
magnetized capacitive RF argon discharge and found that a small magnetic field transverse to the electric field will induce a mode transition from a pressure-heating dominated state to an Ohmic-heating dominated state. You et al. [41, 42] experimentally investigatêd the influence of a magnetic field on the electron power absorption and found that the B-field has a different effect on the power absorption of low-energy electrons at high and low pressures. Electric field reversals during the local sheath collapse are known to affect the electron power absorption in vicinity of the electrodes significantly in CCPs under distinct discharge conditions. In unmagnetized CCPs such electric field reversals have been studied experimentally and computationally [43-45]. They occur, whenever the electron transport to the electrode by diffusion during the local sheath collapse is insufficient to balance the positive ion flux to this electrode on time average. At low pressures such electric field reversals will be caused by the electron inertia, if the sheath collapses so quickly that electrons cannot follow it. At high pressures, field reversals will be caused by collisions, which limit the electrons' movement to the electrodes. In recent studies of the effects of realistic secondary electron emission coefficients (SEECs) on CCP discharges, such electric field reversals were also found to be induced by the strong emission of secondary electrons from boundary surfaces [46]. Similar to previous findings of Campanell et al. [47, 48] a strong secondary electron emission (SEE) leads to a decrease of the effective electron flux to the electrode. In these investigations of electric field reversals in CCPs, electrons were found to gain additional energy due to the acceleration by the reversed electric field, which contributes to the electron power absorption. In magnetized CCP discharges, an 'inverted potential', i.e. a potential at the surface that is positive with respect to that in the plasma bulk, was observed both in experiments and simulations by Kushner et al. [49, 50]. Such inverted potentials were demonstrated to change the charged particle distributions at boundary surfaces. In the investigations of Sharma et al. in helium discharges [24], the inverted potential induced by a small transverse magnetic field was found to lead to an axial asymmetry of the plasma density. Despite the significant influence of these inverted potentials, i.e. electric field reversals induced by the presence of magnetic fields, on the discharge characteristics, the mechanisms of their generation and their effects on the electron dynamics have not been reported and require further clarification.

Previous studies on magnetized CCPs mainly focused on simple atomic electropositive gases. However, complex electronegative gases are used in a number of plasma processing appli- 
cations. CCPs generated in electronegative gases (e.g. oxygen) are characterized by complicated chemical reactions and the presence of negative ions in the discharge plasma. The effect of the magnetic field on the discharge characteristics in such systems has rarely been investigated. In this work, fully kinetic Particle-in-Cell / Monte Carlo collisions (PIC/MCC) simulations are performed to study the influence of a magnetic field on capacitive oxygen plasmas. The simulations reveal that by increasing the magnetic field, that is parallel to the electrodes, a transition of the electron power absorption mode from the DA-mode at low magnetic fields to the $\alpha$-mode can be induced in oxygen CCPs. This effect is based on the variation of the discharge electronegativity (the ratio of the negative ion density to the electron density) as a function of the applied magnetic field and is similar to the mode transitions induced by the variation of the driving voltage waveform, the gas pressure, and gap distance in oxygen CCPs $[28,33,36,37]$. We explain the reasons for the significantly decreased electronegativity of the discharge at stronger magnetic fields based on the dynamics of chemical reactions. Applying a magnetic field leads to an increase of the electron and positive ions densities in the discharge, due to the enhancedionization by electrons in magnetized plasmas. The increase of the electron and positive ion densities is also accompanied by an increased attachment rate. As in the meantime the loss of the negative ions (due to associative detachment) increases at nearly the same rate as the production rate (due to electron impact attachment) the negative ion density is nearly the same independent of the magnetic field within the range studied. In addition, it is found that the presence of the magnetic field leads to the generation of a strong electric field reversal. We apply the Boltzmann-term analysis method $[43,51,52]$ to explain the reason for the generation of such field reversals in magnetized CCPs and to capture the effect of the magnetic field on the electron power absorption. In particular, we show that the reversed field is responsible for an important part of the absorbed power. Further, we present new results for the interaction time of electrons with the expanding sheath edge as a function of the magnetic field, that is shown to result in substantial modifications of the electron energy distribution function. Although illustrated under specific discharge conditions, these findings represent significant new insights into the operation and control of CCPs, which are of general relevance for fundamental research as well as applications.

This paper is structured in the following way: in section 2, we provide a description of the discharge model and its computational implementation. The 'Boltzmann term analysis' 
model is described in section 3. The simulation results are presented in section 4. Finally, concluding remarks are given in section 5 .

\section{PLASMA MODEL AND SIMULATION}
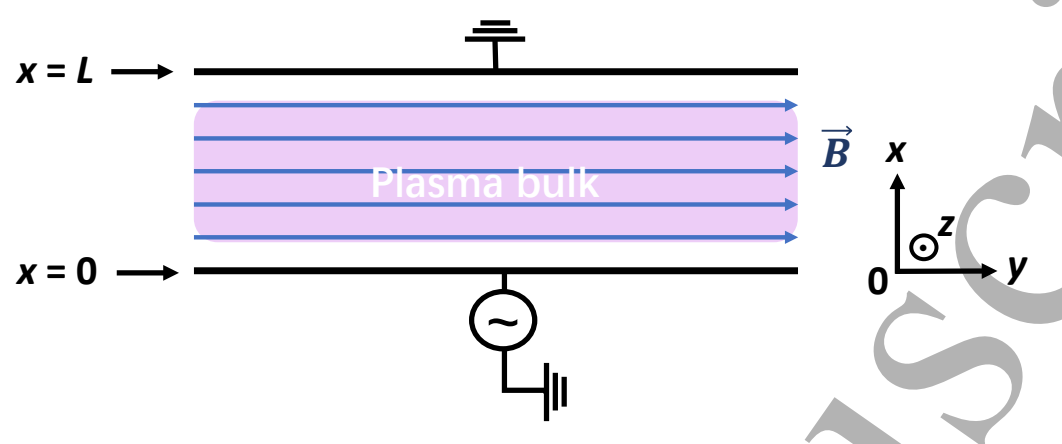

FIG. 1: Schematic of a symmetric capacitive RF discharge in the presence of a uniform magnetic field parallel to the electrodes.

In this work, we employ a $1 \mathrm{~d} 3 \mathrm{v}$ (one-dimensional in space and three-dimensional in velocity space) electrostatic Particle-in-Cell simulation coupled with a Monte Carlo treatment of collision processes. A schematic of the discharge described by our simulation is shown in figure 1. The plasma is operated between two plane parallel electrodes separated by a gap of $L=2.5 \mathrm{~cm}$. The bottom electrode $(x=0)$ is driven by the following voltage waveform:

$$
V(t)=V_{0} \cos (2 \pi f t)
$$

while the top electrode (located at $x=L$ ) is grounded. The electric field $\mathbf{E}$ is directed along the $x$ axis. An axially uniform magnetic field with $\mathbf{B}$ directed along the $y$ axis (i.e. parallel to the electrodes) is present.

The collision processes in oxygen discharges are relatively complicated and several different reaction and cross section sets have been proposed in previous works [53, 54]. In our code, we trace electrons as well as $\mathrm{O}_{-}^{-}$and $\mathrm{O}_{2}^{+}$ions. The reactions and cross sections we implemented in our code can be found in [55]. Metastable $\mathrm{O}_{2}\left(\mathrm{a}^{1} \Delta_{\mathrm{g}}\right)$ molecules have been found to play an important role in oxygen discharges. The electron detachment from $\mathrm{O}^{-}$by collisions with $\mathrm{O}_{2}\left(\mathrm{a}^{1} \Delta_{\mathrm{g}}\right)$ has been demonstrated to be an important loss channel of $\mathrm{O}^{-}$ions. Several previous studies have pointed out that the $\mathrm{O}_{2}\left(\mathrm{a}^{1} \Delta_{\mathrm{g}}\right)$ density greatly influences the charged 
particle power absorption dynamics [37, 56]. A high $\mathrm{O}_{2}\left(\mathrm{a}^{1} \Delta_{\mathrm{g}}\right)$ content in the discharge usually results in a low electronegativity. In our simulations, the $\mathrm{O}_{2}\left(\mathrm{a}^{1} \Delta_{\mathrm{g}}\right)$ particles are assumed to be distributed homogeneously in the chamber. Their density is determined by the same method as used in reference [53], i.e. it is determined from a balance equation. The surface quenching probability of the metastables, $\alpha$, is an important parameter in the simulations, which strongly influences the density of $\mathrm{O}_{2}\left(\mathrm{a}^{1} \Delta_{\mathrm{g}}\right)$ and, therefore, affects the charged particle density significantly. It has been pointed out that the value of $\alpha$ depends on the surface material and temperature $[37,56,57]$. In our simulations, we use $\alpha=6 \times 10^{-3}$, which is identical to the value used in $[28,53,58]$ and has been verified by several experiments to be reasonable.

The equation of motion for a charged particle for a given electric and magnetic field is:

$$
\begin{aligned}
m \frac{d \mathbf{v}}{d t} & =q(\mathbf{E}+\mathbf{v} \times \mathbf{B}) \\
\mathbf{v} & =\frac{d \mathbf{r}}{d t},
\end{aligned}
$$

where $m$ and $q$ are the mass and charge of the particle and $\mathbf{v}$ and $\mathbf{r}$ are its velocity and position. For the calculation of the Lorentz force in the code, we use the Boris rotation [59]. It is worth noting that, in contrast to unmagnetized CCPs where the electron conduction current flows along the electric field lines, an $\mathbf{E} \times \mathbf{B}$ drift of the charged particles is present in magnetized CCPs. In experiments, such a drift motion can be directed towards the reactor walls, where particles interact with boundary surfaces and can be absorbed by the sidewall, which can affect the plasma [60]. For reactors, where the sidewalls are located close to the axial center, this can result in unrealistic results of $1 \mathrm{~d} 3 \mathrm{v}$ PIC/MCC simulations. However, in many experiments this is not the case, i.e. the sidewalls are located far away from the discharge center. Our $1 \mathrm{~d} 3 \mathrm{v}$ code is, therefore, applicable to such scenarios. In addition to this criterion the electrode radius needs to be much larger than the electrode gap to ensure the validity of a $1 \mathrm{~d} 3 \mathrm{v} \mathrm{PIC} / \mathrm{MCC}$ simulation. In such scenarios, the transport of charged particles to the sidewalls is less important and the plasma can still be assumed to be uniform in the $\mathbf{E} \times \mathbf{B}$ direction. Additionally, in our simulations, the velocity of the charged particles is calculated in three dimensions, for which, the $\mathbf{E} \times \mathbf{B}$ drift is considered correctly. Actually, several investigations of such scenarios have been performed based on 1d3v PIC/MCC simulations before [17, 24, 40, 61] and have provided a better understanding of magnetized CCP electropositive discharges. 


\section{MODEL OF THE ELECTRIC FIELD GENERATION}

The spatio-temporal electron power absorption dynamics has recently been investigated based on the momentum balance equation, i.e. the first velocity moment of the Boltzmann equation, in unmagnetized capacitive plasma discharges [43, 51, 52]. Here, we extend this "Boltzmann term analysis" to magnetized capacitive discharges, in order to understand the generation of electric field reversals.

Under the conditions studied in this work the term of the electron momentum balance that depends on ionization is negligible. The momentum balance equation for electrons is then:

$$
m\left[\frac{\partial(n \mathbf{u})}{\partial t}+\nabla \cdot(n \mathbf{u u})\right]=-e n(\mathbf{E}+\mathbf{u} \times \mathbf{B})-\nabla \cdot \mathbf{P}-\mathbf{\Pi} .
$$

Here, $n$ and $\mathbf{u}=\left\{u_{\mathrm{x}}, u_{\mathrm{y}}, u_{\mathrm{z}}\right\}$ are the electron density and mean velocity in three directions, respectively, $m$ is the electron mass, and $\mathbf{P}$ is the electron pressure tensor. $\boldsymbol{\Pi}$ represents the effective electron momentum loss due to collisions per volume and time. As described in section 2, the magnetic field, $\mathbf{B}=\left\{0, B_{\mathrm{y}}, 0\right\}$, is parallel to the electrodes and the electric field, $\mathbf{E}=\left\{E_{\mathrm{x}}, 0,0\right\}$, is normal to the electrodes. Taking the momentum balance in the $x$ 
direction (perpendicular to the electrodes) yields:

$$
m \frac{\partial\left(n u_{\mathrm{x}}\right)}{\partial t}+m \frac{\partial\left(n u_{\mathrm{x}}^{2}\right)}{\partial x}=-e n\left(E_{\mathrm{x}}-u_{\mathrm{z}} B_{\mathrm{y}}\right)-\frac{\partial p_{\mathrm{xx}}}{\partial x}-\Pi_{\mathrm{x}}
$$

where $p_{\mathrm{xx}}=n T_{\mathrm{xx}}$ denotes the diagonal element of the pressure tensor and $T_{\mathrm{xx}}=m\left(<v_{\mathrm{x}}^{2}>\right.$ $\left.-u_{\mathrm{x}}^{2}\right)$, where $v_{\mathrm{x}}$ is the velocity of an individual electron in the $x$-direction normal to the electrodes. It is worth noting that some shear terms of the pressure tensor are not zero, since $u_{z} \neq 0$. However, in our $1 \mathrm{~d} 3 \mathrm{v}$ simulation, $\frac{\partial}{\partial y}(\ldots)=\frac{\partial}{\partial z}(\ldots)=0$ and, thus, $\frac{\partial}{\partial x}\left(p_{\mathrm{xx}}\right)$ is the only non-zero contribution from the pressure tensor in the x-component of the/momentum balance equation. Finally, we rearrange equation (4) and replace $E_{\mathrm{x}}$ by $E_{\text {model }}$ :

$$
E_{\text {model }}=E_{\text {in }}+E_{\nabla \mathrm{P}}+E_{\mathrm{Ohm}}+E_{\mathrm{B}}
$$

where

$$
\begin{aligned}
& E_{\mathrm{in}}=-\frac{m}{e n}\left(\frac{\partial\left(n u_{\mathrm{x}}\right)}{\partial t}+\frac{\partial\left(n u_{\mathrm{x}}^{2}\right)}{\partial x}\right) \\
& E_{\nabla \mathrm{P}}=-\frac{1}{e n} \frac{\partial\left(n T_{\mathrm{xx}}\right)}{\partial x} \\
& E_{\mathrm{Ohm}}=-\frac{\Pi_{\mathrm{x}}}{e n} \\
& E_{\mathrm{B}}=u_{\mathrm{z}} B_{\mathrm{y}}
\end{aligned}
$$

Thus, we decompose the total electric field into four different terms, the inertial term, $E_{\text {in }}$, the pressure term, $E_{\nabla \mathrm{P}}$, the collision term, $E_{\mathrm{Ohm}}$, as well as the magnetically induced term (Lorentz force term), $E_{\mathrm{B}}$. Each of these terms is associated with a distinct physical mechanism of electric field generation. Moreover, multiplying each electric field term with the electron conduction current density yields the electron power absorption due to the respective mechanism. For instance, for $E_{\mathrm{Ohm}}$ this multiplication yields the Ohmic electron power absorption, while for $E_{\nabla \mathrm{P}}$ it yields the electron pressure heating.

We will see later that the pressure term has a significant contribution to the total electric field. It is also worth noting that the mean electron velocity in the $z$-direction, $u_{\mathrm{z}}$, is induced by the $\mathbf{E} \times \mathbf{B}$ drift and is responsible for the generation of the electric field reversals during sheath collapse at each electrode, i.e. there is a magnetically induced electric field reversal. In combination with the presence of collisions with neutrals it also enhances the Ohmic electron power absorption significantly [62].

The time and space resolved input data for this model are taken from the PIC/MCC simulations. These are the electron density, mean electron velocity, as well as the random thermal 
electron velocity and the electron momentum loss. These input parameters are substituted into equation (6) to analyse and understand the formation of the electric field space and time resolved within the $\mathrm{RF}$ period.

\section{RESULTS}
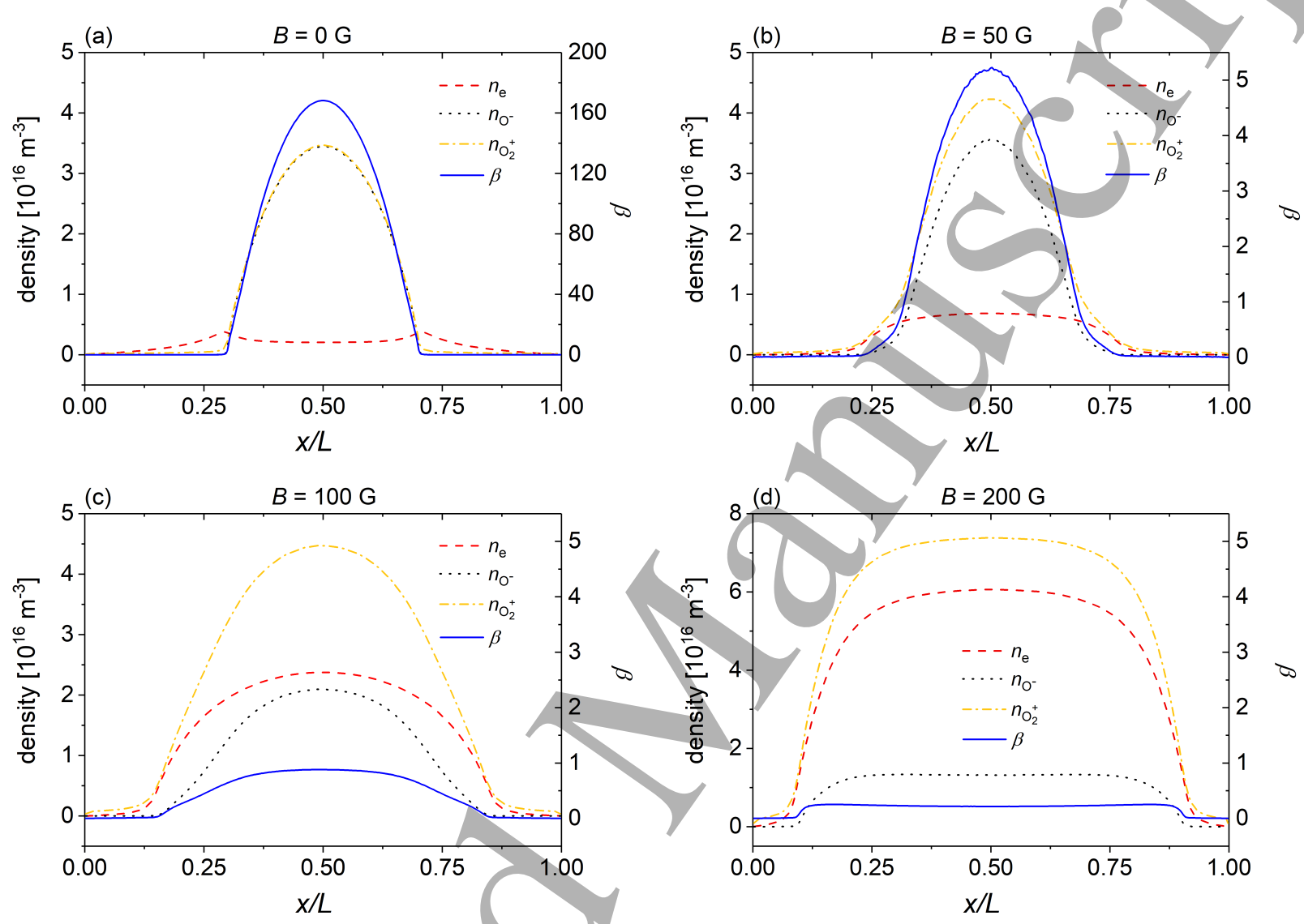

FIG. 2: Profiles of the time ayeraged electron density, $\mathrm{O}^{-}$density, $\mathrm{O}_{2}^{+}$density, and electronegativity, $\beta$, for different magnetic field strengths of $B=0 \mathrm{G}(\mathrm{a}) ; B=50 \mathrm{G}(\mathrm{b}) ; B=100 \mathrm{G}$ (c); and $B=200 \mathrm{G}(\mathrm{d})$. Discharge conditions: $L=2.5 \mathrm{~cm}, p=100 \mathrm{mTorr}, f=13.56 \mathrm{MHz}$, and $V_{0}=300 \mathrm{~V}$.

First, we investigate the effect of the magnetic field on the charged particle density profiles in oxygen discharges. Figure 2 shows the time averaged densities of electrons, $\mathrm{O}^{-}$and $\mathrm{O}_{2}^{+}$ions, as well as the electronegativity $\beta=n_{\mathrm{O}^{-}} / n_{\mathrm{e}}$ at magnetic field strengths of $B=$ 0,50,100,200 G. As specified earlier, the discharge is operated at $13.56 \mathrm{MHz}$ with a driving voltage amplitude of $300 \mathrm{~V}$ at a pressure of $100 \mathrm{mTorr}$ and an electrode gap of $2.5 \mathrm{~cm}$. In the 
absence of a magnetic field, i.e. at $B=0 \mathrm{G}$, the electronegativity is high with a peak value of around 170 in the center. The electron density profile is depleted in the electronegative bulk, but peaks appear in the electropositive edge region of the discharge close to the positions of maximum sheath width at each electrode.

If a transverse magnetic field of $B=50 \mathrm{G}$ is applied, as shown in figure 2(b), the peak electron density is greatly increased and appears at the discharge center, while the peak density of $\mathrm{O}^{-}$ions remains almost unchanged. As a result, the electronegativity is greatly reduced to about 5. When the magnetic field is increased to $100 \mathrm{G}$ and $200 \mathrm{G}$, the electron and $\mathrm{O}_{2}^{+}$ion densities are further increased along with a large reduction of the peak $\mathrm{O}^{-}$ density and the electronegativity. As shown in figure 2(d), the electronegativity is further decreased to about 0.2 in the bulk region at $B=200 \mathrm{G}$. Due to the enhanced electron density, the sheath width is reduced. While the peak density of $\mathrm{O}^{-}$ions varies with $B$, their time and space averaged density, as it will be shown later, remains almost the same at any $B$.

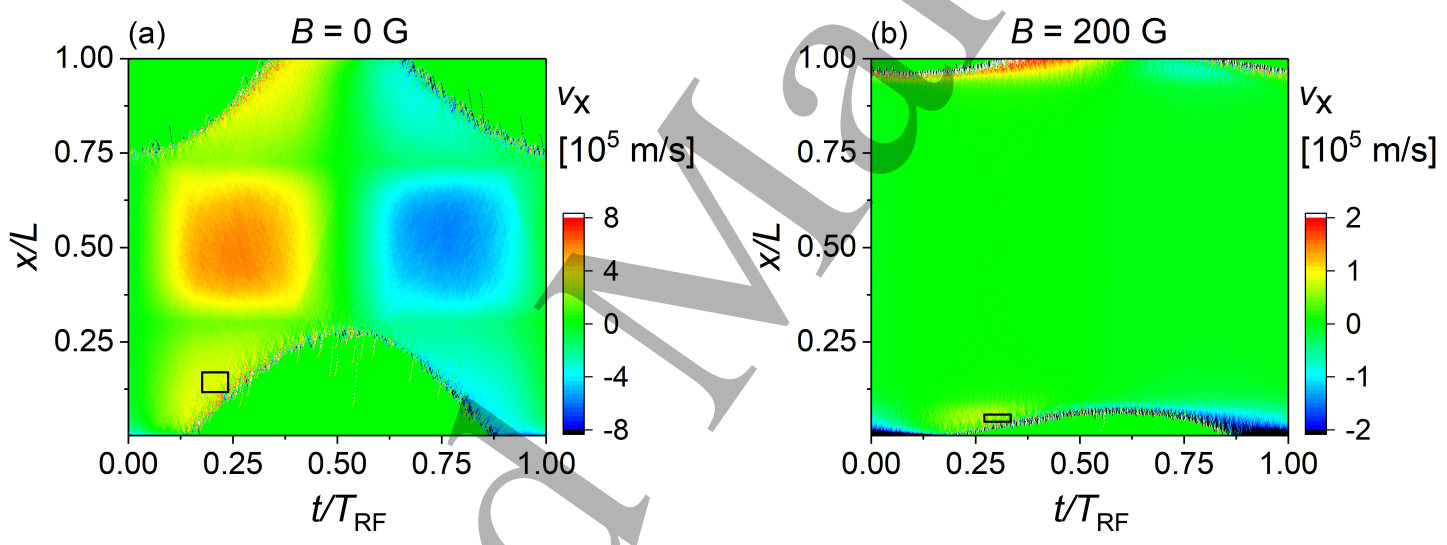

FIG. 3: Spatio-temporal plots of the electron velocity in axial direction $v_{x}$ at $B=0 \mathrm{G}$ (a) and $B=200 \mathrm{G}(\mathrm{b})$. Discharge conditions: $L=2.5 \mathrm{~cm}, p=100 \mathrm{mTorr}, f=13.56 \mathrm{MHz}$, and $V_{0}=300 \mathrm{~V}$. The black rectangles (regions of interest, ROIs) aid the estimation of the interaction time of electrons with the expanding sheath edge (see text). The powered electrode is located at $x / L=0$, while the grounded electrode is located at $x / L=1$.

The observed increase of the electron density as a function of the magnetic field is explained by the combination of three effects. Firstly, the presence of a magnetic field parallel to the electrodes enhances the electron confinement and, thus, the plasma density.

Secondly, the magnetic confinement of electrons close to the oscillating RF sheaths results in 
an increase of the interaction time of these electrons with the oscillating boundary sheaths. Figure 3 shows the spatio-temporal distribution of the electron velocity in the axial direction for $B=0 \mathrm{G}$ and $B=200 \mathrm{G}$. To verify the extended interaction time of electrons with the expanding sheath edge due to the presence of the magnetic field, we estimate this interaction time by dividing the maximum sheath width by the average electron velocity in $x$ direction within the regions of interest (ROI) indicated in figure 3 by the black rectangles. These ROIs are chosen to include the spatio-temporal regions of maximum axial/electron velocity during sheath expansion. For $B=0 \mathrm{G}$ in figure $3(\mathrm{a})$, the maximum sheath width is $6.36 \times 10^{-3} \mathrm{~m}$. The average electron velocity in axial direction within the spatio-temporal ROI is $3.25 \times 10^{5} \mathrm{~m} / \mathrm{s}$. Therefore, the interaction time of electrons with the expanding sheath is about $19.6 \mathrm{~ns}$. For $B=200 \mathrm{G}$, the electron velocity in $x$ direction is decreased. In this case, the maximum sheath width is about $2.1 \times 10^{-3} \mathrm{~m}$ and the average value of $v_{x}$ within the spatio-temporal ROI indicated in figure $3(\mathrm{~b})$ is about $4.4 \times 10^{4} \mathrm{~m} / \mathrm{s}$. The interaction time of the electrons and the expanding sheath then is about $47.7 \mathrm{~ns}$ for this magnetized scenario. Therefore, due to the magnetic confinement, the interaction time of electrons with the expanding sheath is enhanced and electrons can/be accelerated by the expanding sheath for a longer time within a given RF period. This enhances the electron power absorption and finally contributes to the increase of the plasma density.

Finally, the increased electron density is also related to the generation of a strong electric field reversal during the sheath collapse in the presence of the magnetic field. Such electric field reversals directly enhance the electron power absorption. This phenomenon of a magnetically enhanced electric field reversal will be investigated in more detail below. 

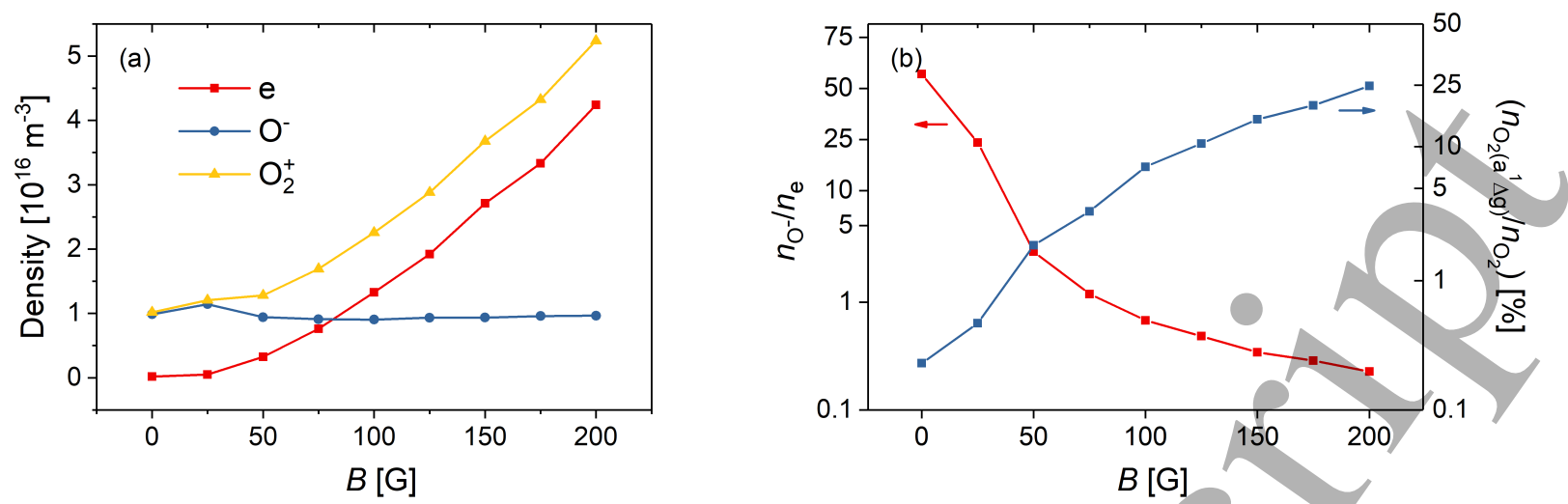

FIG. 4: Space and time averaged electron, $\mathrm{O}^{-}$and $\mathrm{O}_{2}^{+}$density (a), electronegativity and ratio of the $\mathrm{O}_{2}\left(\mathrm{a}^{1} \Delta_{\mathrm{g}}\right)$ density and the $\mathrm{O}_{2}$ density (b) as a function of the magnetic field magnitude. Discharge conditions: $L=2.5 \mathrm{~cm}, p=100 \mathrm{mTorr}, f=13.56 \mathrm{MHz}$, and $V_{0}=300 \mathrm{~V}$.

Figure 4(a) shows the space and time averaged electron and ion densities as a function of the magnetic field strength. For the reasons described above the electron and $\mathrm{O}_{2}^{+}$density increase as a function of the magnetic field. However, the space and time averaged $\mathrm{O}^{-}$density remains approximately constant, although its peak density decreases in the discharge center, but the width of the region of high negative ion density is increased due to the decrease of the sheath width as a function of the magnetic field (see figure 2). Consequently, the space and time averaged electronegativity is decreased from 58 to 0.23 with increasing magnetic field, as shown in figure 4(b). The electronegativity values that we find at low magnitudes of $\mathrm{B}$ are higher than those observed previously by other authors. The origin of these differences may be sought (i) in the different materials used for the electrodes and other parts of the plasma chamber in various experiments and (ii) in the operating conditions, especially the power level. (i) The materials, via the surface quenching coefficient of the oxygen singlet delta metastable molecules can have a dramatic effect on the plasma chemistry and in turn have a major influence on the plasma characteristics, like the electronegativity. It has been reported both by experiments and simulations that a high quenching coefficient of $\mathrm{O}_{2}\left(\mathrm{a}^{1} \Delta_{\mathrm{g}}\right)$ molecules can lead to a high $\mathrm{O}^{-}$density and high discharge electronegativity $[28,56,57]$. Previous measurements, e.g. by Katsch et al. [30], Stoffels et al. [63] and Kuellig et al. [64] concluded in electronegativity values between 1 and 10. In the measurements of Kaga et al. [65] values up to 20 were found. (ii) Most of the above experiments have been conducted at power density levels much higher than those used by Derzsi et al $[28,36]$ and showed 
strong indications that the electronegativity increases towards lower power densities. This was also found to be the case by Gudmundsson et al. [66]. In addition, previous studies also indicate that both the decreasing gap size [39] and gas pressure [33] can lead to an increased electronegativity and electron power absorption mechanisms specific of the DAmode. Considering these, the high electronegativities obtained from our calculations do not actually contradict the findings of previous works. At the same time, our results have as well some uncertainty, that is expected to be higher than simulation results for similar/systems in atomic gases, due to the complexity of the plasma chemistry in molecular gases, which makes it difficult to build accurate models, and due to the uncertainty of the data available for the elementary processes.

The ratio of the metastable $\mathrm{O}_{2}\left(\mathrm{a}^{1} \Delta_{\mathrm{g}}\right)$ and the $\mathrm{O}_{2}$ densities at different strength of the magnetic field is shown in figure 4(b). Due to the increase of the electron density and the magnetic electron confinement, the number of collisions between electrons and $\mathrm{O}_{2}$ molecules is enhanced. Therefore, more $\mathrm{O}_{2}\left(\mathrm{a}^{1} \Delta_{\mathrm{g}}\right)$ metastables are generated by electron impact excitation at high magnetic fields. This increase of the $\mathrm{O}_{2}\left(\mathrm{a}^{1} \Delta_{\mathrm{g}}\right)$ metastable content is one of the main reasons for the almost unchanged $\mathrm{O}^{-}$(density, since it is the basis of the dominant loss channel of $\mathrm{O}^{-}$ions via associative electron detachment $\left(\mathrm{O}^{-}+\mathrm{O}_{2}\left(\mathrm{a}^{1} \Delta_{\mathrm{g}}\right) \rightarrow \mathrm{O}_{3}+\mathrm{e}^{-}\right)$. While the rate of the electron detachment, that represents a loss of negative ions, increases as a function of the magnetic field, the source of $\mathrm{O}^{-}$ions via dissociative electron attachment $\left(\mathrm{e}^{-}+\mathrm{O}_{2} \rightarrow \mathrm{O}^{-}+\mathrm{O}\right)$ also increases as a function of the magnetic field in the same way. As we will see below, the increase of the electron density as a function of the magnetic field is the primary reason for this effect. 

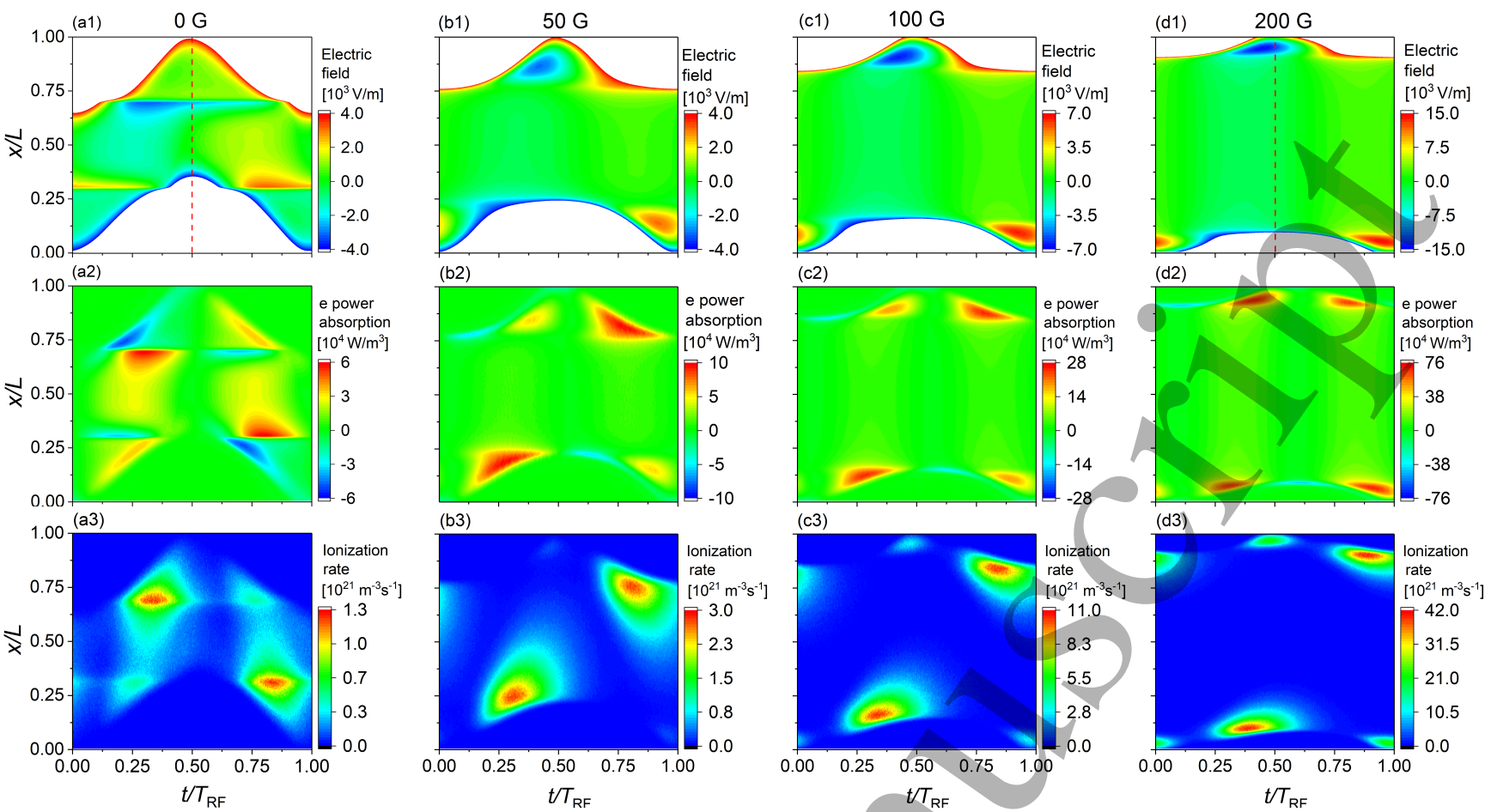

FIG. 5: Spatio-temporal plots of the electric field (first row), the electron power absorption rate (second row), and the ionization rate (third row) at magnetic fields of $B=0 \mathrm{G}$ (first column), $B=50 \mathrm{G}$ (second column), $B=100 \mathrm{G}$ (third column), and $B=200 \mathrm{G}$ (fourth column). The vertical dashed lines in (a1) and (d1) indicate the time of sheath collapse at the top electrode, which will be further analyzed to understand the generation of electric field reversals (see figure 7). The powered electrode is located at $x / L=0$, while the grounded electrode is located at $x / L=1$. Discharge conditions: $L=2.5 \mathrm{~cm}, p=100$ mTorr, $f=13.56 \mathrm{MHz}$, and $V_{0}=300 \mathrm{~V}$.

Figure 5 shows the spatio-temporal distributions of the electric field, the electron power absorption rate, and the iomzation rate for different magnetic fields. At $B=0 \mathrm{G}$, the discharge is strongly electronegative and the electron density as well as the conductivity in the plasma bulk region are low. Thus, a drift electric field is generated in the bulk at the times of maximum RF current. At the same time, strong ambipolar electric fields are generated at the positions of largest electron density gradient close to the positions of maximum sheath width at each electrode due to the formation of electropositive edge regions. Electrons are accelerated by these drift and ambipolar electric fields. In this way maxima of the ionization rate show up at the positions of strongest ambipolar electric field close to the sheath edge during its collapse phase, as shown in figure 5(a3). Another ionization peak appears near the 
expanding sheath, which is created by electrons that have been accelerated during sheath expansion. In this case, the discharge is operated in the DA mode.

If a magnetic field of $B=50 \mathrm{G}$ is applied, the electron density and, thus, the conductivity increase in the bulk region. Therefore, the field field vanishes inside the bulk. Under these conditions, electrons are mainly accelerated close to the instantaneous sheath edge during sheath expansion. Simultaneously, an electric field reversal appears at each electrode during the local sheath collapse. Figure 5(b2) shows that this field reversal causes significant electron power absorption. If the magnetic field is increased to $100 \mathrm{G}$ and $200 \mathrm{G}$, the sheath width decreases significantly and the electric field reversal gets stronger. Maxima of the ionization rate are generated at the positions where the field reversal appears. The electron power absorption rate is higher in the regions of the reversed electric field during sheath collapse compared to the sheath expansion phase at $B=200 \mathrm{G}$. Thus, the electric field reversal is essential for the generation of the discharge under these conditions. Although the electron power absorption by the electric field reversal is stronger compared to the electron power absorption during the sheath expansion phase in figure $5(\mathrm{~d} 2)$, the ionization rate is higher during sheath expansion, as shown in figure 5(d3). This is caused by several different effects. One reason is that the electron acceleration by the electric field reversal happens close to and towards the adjacent electrode. The accelerated electrons cannot propagate a long distance and cannot cause much ionization before they are absorbed by the electrode. Some of the electrons accelerated by the reversed electric field are reflected by the expanding sheath a few nanoseconds later. These electrons are accelerated by both the electric field reversal and the sheath expansion. As a result, they have higher energies, can propagate a long distance towards the discharge center and, thus, can cause more ionization. For these reasons the ionization rate is maximum during the sheath expansion phase. 

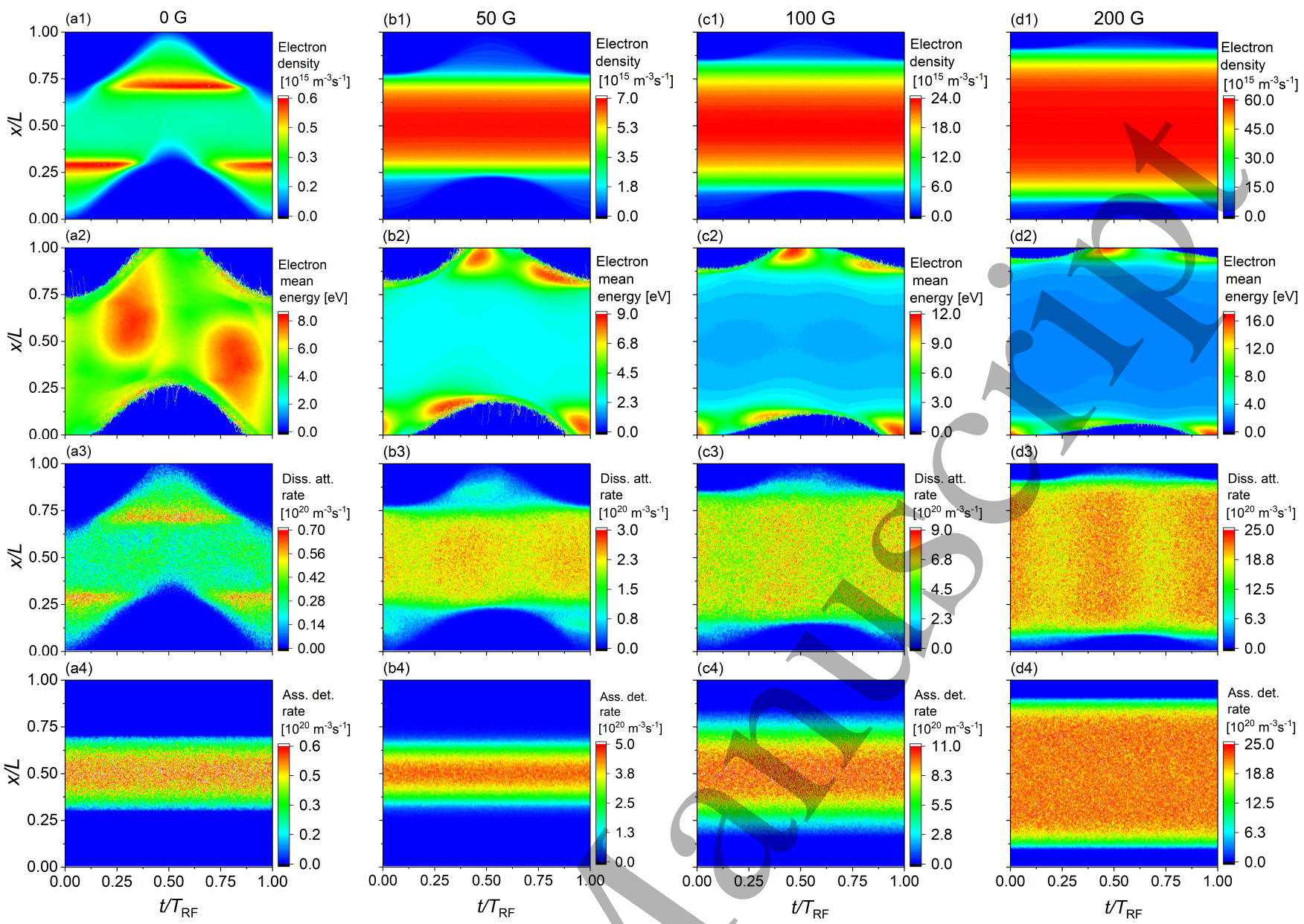

FIG. 6: Spatio-temporal plots of the electron density (first row), the mean electron energy (second row), the dissociative attachment rate (third row), and the associative detachment rate (fourth row) at magnetic fields of $B=0 \mathrm{G}$ (first column), $B=50 \mathrm{G}$ (second column), $B=100 \mathrm{G}$ (third column), and $B=200 \mathrm{G}$ (fourth column). The powered electrode is located at $x / L=0$, while the grounded electrode is located at $x / L=1$. Discharge conditions: $L=2.5 \mathrm{~cm}, p=100$ mTorr, $f=13.56 \mathrm{MHz}$, and $V_{0}=300 \mathrm{~V}$.

To clarify the reason for the constant space and time averaged $\mathrm{O}^{-}$density as a function of the magnetic field, we analyze the sources and the losses of $\mathrm{O}^{-}$in the discharge and the changes of these collision rates as a function of the magnetic field in more detail. In the simulations, the dissociative attachment is the only source of $\mathrm{O}^{-}$ions. The loss of $\mathrm{O}^{-}$is due to four different reactions in our code (see [55]). Under the conditions studied here, the associative electron detachment is the main loss channel of $\mathrm{O}^{-}$causing more than $80 \%$ of the total loss at $B=0 \mathrm{G}$ and to an even higher percentage at $B>0 \mathrm{G}$. Figure 6 shows the spatio-temporal 
distributions of the electron density, the mean electron energy, and the dissociative electron attachment as well as the associative electron detachment rate as a function of the magnetic field. With the increase of the magnetic field, the electron density is increased significantly. The density peak moves from the sheath edge to the discharge center. The spatio-temporal distribution of the mean electron energy changes drastically as a function of the magnetic field. For $B=0 \mathrm{G}$, the highest electron energy is observed in the plasma bulk, where the electrons are accelerated by the high drift electric field in the DA mode. At $B \Leftarrow 50 \mathrm{G}$, the electron energy peaks close to the electrodes during the local sheath expansion and collapse phases due to the electron acceleration by the expanding sheath and the electric field reversal, respectively. At the highest magnetic field, the electron energy is maximum at the electrodes during the local sheath collapse due to the strong magnetically induced electric field reversal. These results also verify that the mean electron energy increases near the expanding sheath edge as a function of the externally applied magnetic field, which is attributed to a longer interaction time between the electrons and the expanding sheath at high magnetic fields.

Although the mean electron energy changes significantly as a function of the magnetic field, the dissociative attachment rate mainly follows the variation of the electron density, i.e. the dissociative attachment is significantly enhanced and the maximum of the dissociative attachment rate is shifted from the edge to the discharge center by increasing the magnetic field. Due to the electron power absorption caused by the electric field reversal, very high electron energies are found close to the electrodes during the local sheath collapse at high magnetic fields. Only at $B=50 \mathrm{G}$ a local maximum of the dissociative attachment rate is observed at this position. At $B=100 \mathrm{G}$ and $B=200 \mathrm{G}$ most of the electrons are too energetic within this spatio-temporal region after being accelerated by the strong reversed electric field. The cross section for dissociative attachment initially increases as a function of the electron energy, reaches its maximum at about $6.5 \mathrm{eV}$ and then decreases. Due to the strong acceleration by the electric field reversal, most of these electrons have a high energy, which results in a low dissociative attachment probability. At the same time, the electron density is very low at this position. Thus, the dissociative attachment is much lower at this position compared to that near the discharge center. As the $\mathrm{O}^{-}$and metastable densities and energies are essentially constant as a function of time, the associative detachment rate remains approximately constant in time, too. As a result of the increased $\mathrm{O}_{2}\left(\mathrm{a}^{1} \Delta_{\mathrm{g}}\right)$ density 
and the decreased sheath width at high magnetic fields, the associative detachment rate is much higher at large B-fields and is significant over a much larger fraction of the electrode gap. Both, the sources and losses of $\mathrm{O}^{-}$ions, increase as a function of the externally applied magnetic field. Our analysis shows that these increases are ultimately caused by the higher electron density. Changes of the mean electron energy play a less important role. As both rates increase similarly as a function of the magnetic field, their balance results in an almost unchanged $\mathrm{O}^{-}$density. Simultaneously, the positive ion density, however, increases and, thus, the electronegativity decreases.
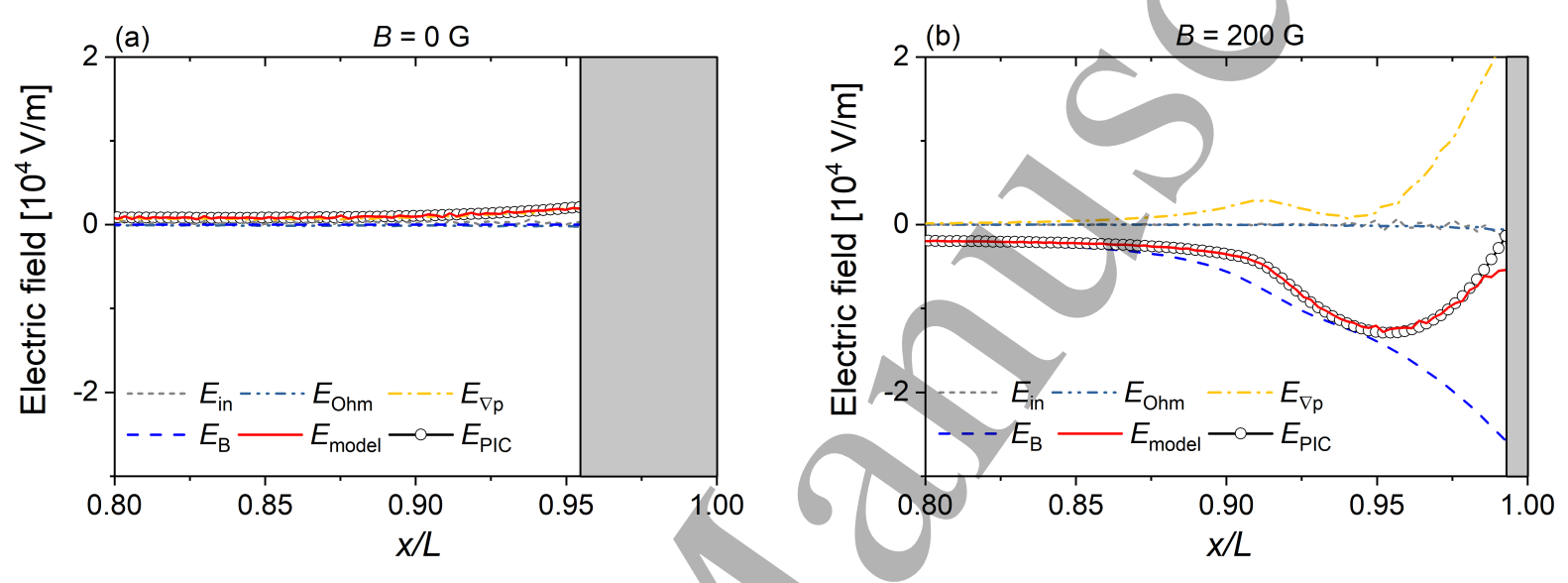

FIG. 7: Profiles of the electric field terms obtained from the Boltzmann term model and the electric field obtained directly from the PIC simulation in the vicinity of the grounded electrode at $B=0 \mathrm{G}(\mathrm{a})$, and $B=200 \mathrm{G}(\mathrm{b})$ at the time of sheath collapse $\left(t=0.5 T_{\mathrm{RF}}\right)$ indicated by the vertical dashed lines in figure 5 . Discharge conditions: $L=2.5 \mathrm{~cm}, p=100$ mTorr, $f=13.56 \mathrm{MHz}$, and $V_{0}=300 \mathrm{~V}$. The gray rectangles indicate the sheath region, where the electron density is extremely low and no model results can be obtained. The sheath edge is calculated by the Brinkmann-criterion [67]. The sum of the terms, $E_{\text {model }}$, reproduces well the electric field computed from the simulation, $E_{\mathrm{PIC}}$, except in the proximity of the electrode where the low electron density makes the computation of the terms less accurate.

To better understand the generation of the electric field reversals during sheath collapse, we apply the Boltzmann term model based on input parameters obtained from the simulation. Figure 7 shows the axial electric field terms obtained from the model according to equation (6) and the electric field computed from the PIC simulation close to the grounded electrode and at the time of the local sheath collapse $\left(t=0.5 T_{\mathrm{RF}}\right)$, which is indicated by the vertical 
dashed line in figure 5(a1) and (d1), respectively, for two different values of the magnetic field strength. The model reproduces well the electric field profiles obtained from the simulation. At $B=0 \mathrm{G}$, the electric field is low close to this electrode at this time within the RF period, i.e. no electric field reversal is generated. At $B=200 \mathrm{G}$, a strong electric field reversal is, however, observed $(E<0)$. The model now allows to understand its generation by splitting it up into the different terms according to equation (6). The inertia term, $E_{\text {in }}$, and the Ohmic term, $E_{\mathrm{Ohm}}$, are found to be negligible and only the pressure term, $E_{\nabla \mathrm{P}}$, and the/Lorentz force term, $E_{\mathrm{B}}$, contribute significantly to the total electric field under these conditions. $E_{\nabla \mathrm{P}}$ is positive, i.e. it does not cause the electric field reversal, but accelerates electrons towards the bulk. It largely consists of the ambipolar electric field. Thus, the negative $E_{\mathrm{B}}$ term is the only term that produces the electric field reversal. This term is caused by the Lorentz force in the electron momentum balance equation and, thus, the electric field reversal is induced by the presence of the externally applied magnetic field. Based on equation (6) this high value of $E_{B}$ close to the electrode during the local sheath collapse is related to a high electron velocity in $z$-direction parallel to the electrodes, which is the result of the electron $\mathbf{E} \times \mathbf{B}$ drift. Due to the magnetic electron confinement the electron flux to the electrodes is limited. The ions are not magnetized and, thus, their flux to the electrodes is not limited by the presence of the magnetic field. In order to compensate the high ion flux at each electrode on time average in the presence of the magnetic electron confinement, an electric field reversal must be generated to accelerate electrons towards the electrode. Once a small reversed electric field is generated, $u_{z}$ increases due to the $\mathbf{E} \times \mathbf{B}$ drift, which in turn further enhances the electric field reversal, until the ion flux can be compensated by the electron flux at each electrode on time average. 

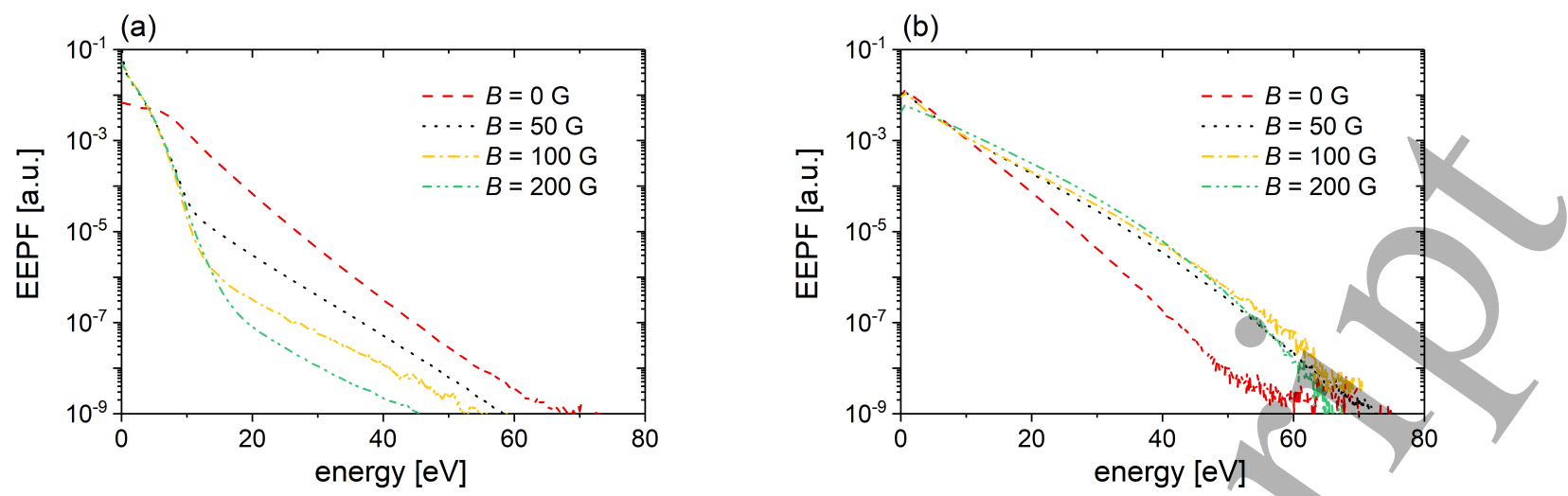

FIG. 8: Time averaged electron energy probability function (EEPF) in the discharge center (a) and local EEPF near the expanding sheath edge averaged over spatio-temporal ROIs indicated in figure 3 (b) for different externally applied magnetic fields. Discharge conditions: $L=2.5 \mathrm{~cm}$, $p=100$ mTorr, $f=13.56 \mathrm{MHz}$, and $V_{0}=300 \mathrm{~V}$.

The time averaged EEPF in the discharge center as a function of the magnetic field is shown in figure $8(\mathrm{a})$. For $B=0 \mathrm{G}$, the EEPF is convex, the number of low energy electrons is low. Under these conditions, the electron power absorption in the bulk region induced by the drift and ambipolar electric fields is dominant, the electronegativity is high, and there is no magnetic electron confinement. Increasing the magnetic field, leads to a decrease of the electronegativity, an enhanced electron confinement, and a power absorption mode transition from the DA-mode to $\alpha$-mode where the electron power absorption is attenuated in the discharge center and strong at the sheath edges. This causes the presence of more low energy electrons in the discharge center. The transport of highly energetic electrons from the oscillating sheath edges into the discharge center is reduced by the presence of the magnetic field. The corresponding low energy part of the EEPF is, therefore, enhanced in the discharge center at high magnetic fields. Figure 8(b) shows the EEPF during the sheath expansion phase as a function of the magnetic field by collecting data from spatiotemporal ROIs near the expanding sheath edges. For $B=0 \mathrm{G}$ and $B=200 \mathrm{G}$, the ROIs are indicated in figure 3. As discussed above, the presence of a large magnetic field enhances the electron heating by confining the electrons near the expanding sheath. Due to the extended interaction time of the electrons and the expanding sheath, more electrons are accelerated to relatively high energies compared to the $B=0 \mathrm{G}$ case, which is an important factor that finally enhances the plasma density in magnetized CCP discharges. 

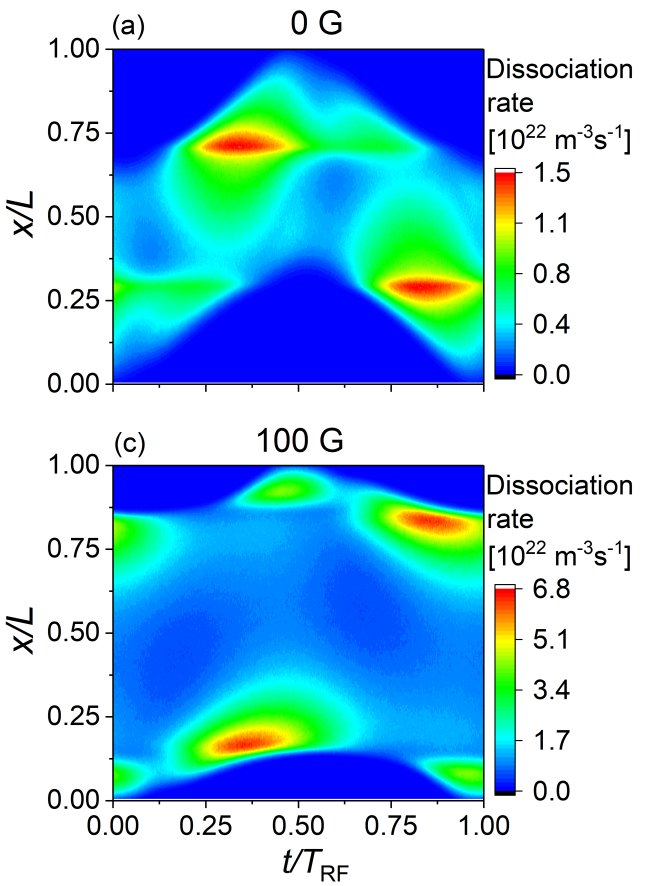
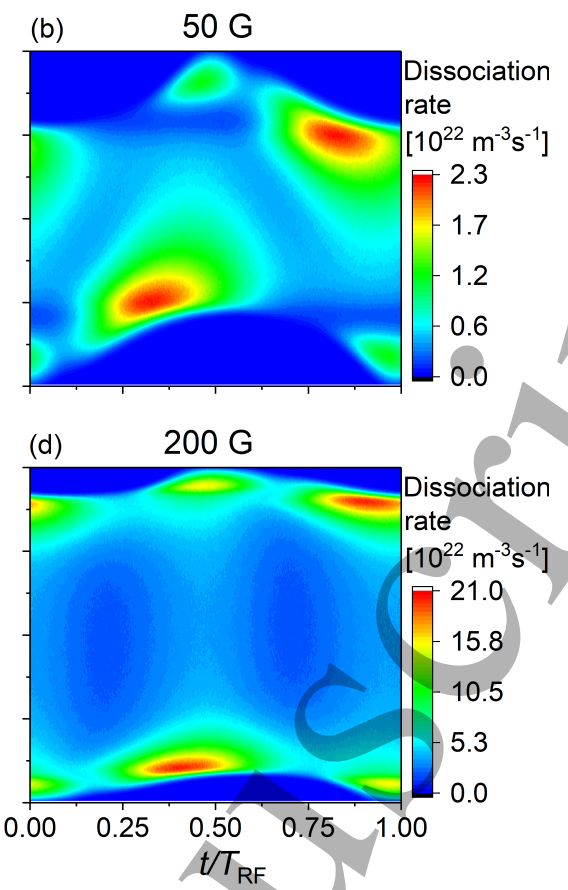

FIG. 9: Spatio-temporal plots of the total dissociation rate of $\mathrm{O}_{2}$ at magnetic fields of $B=0 \mathrm{G}$, $B=50 \mathrm{G}, B=100 \mathrm{G}$, and $B=200 \mathrm{G}$. The powered electrode is located at $x / L=0$, while the grounded electrode is located at $x / L=1$. Discharge conditions: $L=2.5 \mathrm{~cm}, p=100$ mTorr, $f=13.56 \mathrm{MHz}$, and $V_{0}=300 \mathrm{~V}$.

In oxygen discharges, the generation of oxygen atoms and their interaction with boundary surfaces usually play an important role for etching and deposition processes. Thus, the effect of the externally applied magnetic field on the generation of $\mathrm{O}$ atoms is studied. The $\mathrm{O}_{2}$ dissociation rates resulting from the three dissociation reactions included in the PIC simulation (see [55]) are added and the sum is shown as the total dissociation rate in figure 9 for different magnetic field strengths. By increasing the magnetic field, the total dissociation rate is enhanced. Due to the electron power absorption mode transition the peak of the total dissociation rate moves from the position, where the ambipolar field is maximum in the DA-mode at $B=0 \mathrm{G}$, to the expanding sheath edge at high magnetic fields. Moreover, at high B-fields the electric field reversal also enhances the dissociation of oxygen molecules and contributes to the generation of oxygen atoms significantly. Overall, the total dissociation rate of oxygen molecules is strongly correlated with the spatio-temporal dynamics of energetic electrons. 


\section{CONCLUSIONS}

The influence of a uniform externally applied magnetic field ( $0 \leq B \leq 200 \mathrm{G}$ ) parallel to the electrodes on the spatio-temporal electron power absorption dynamics and plasma properties was investigated by PIC/MCC simulations in electronegative oxygen discharges at $13.56 \mathrm{MHz}, 100 \mathrm{mTorr}$, and a constant driving voltage amplitude of $300 \mathrm{~V}$. The presence of the magnetic field enhances the confinement of electrons to the plasma. This leads to a longer interaction time of electrons with the oscillating RF sheaths and, thus, enhances the electron power absorption. Moreover, it causes electrons to undergo more collisions before they are absorbed at the electrodes. Another important effect that leads to an increase of the electron power absorption is the generation of magnetically induced electric field reversals at each electrode during the local sheath collapse.

The change of the electron power absorption dynamics with increasing magnetic field was found to cause an increase of the electron and $\mathrm{O}_{2}^{+}$ion density, while the $\mathrm{O}^{-}$ion density was found to remain approximately constant. This is caused by the spatio-temporal evolution of the dissociative electron attachment and the associative electron detachment rates. These reactions correspond to the source and the main loss channel of $\mathrm{O}^{-}$ions in the discharge. Both rates increase in a similar way due to the increase of the electron density as a function of the externally applied magnetic field. This increase of $n_{e}$ directly causes an increase of the dissociative attachment rate. The associative detachment rate is also enhanced, since the production of $\mathrm{O}_{2}\left(\mathrm{a}^{1} \Delta_{\mathrm{g}}\right)$ metastables increases as a function of the electron density and, thus, of the magnetic field. The increases of these rates are found to be induced by an increase of the electron density rather than changes of the mean electron energy. The balance of these two reactions finally results in a constant spatially averaged $\mathrm{O}^{-}$density for different B-fields.

As a result of the changes of the electron and $\mathrm{O}^{-}$density, the electronegativity decreases as a function of the externally applied magnetic field. This causes an electron power absorption mode transition from the DA-mode to the $\alpha$-mode. As part of this mode transition a reversed electric field is generated during the sheath collapse at each electrode and gets stronger at larger magnetic fields. The generation of this reversed electric field causes significant electron power absorption and ionization as well as dissociation of the molecular background gas.

In order to understand the generation of this electric field reversal, a Boltzmann term analysis 
model was applied, which allowed to split the total electric field into several different terms, each one corresponding to a distinct physical mechanism of electric field generation: electron inertia, pressure gradient, and collisional to magnetic field effects. By substituting the electron density, drift velocity, momentum loss, as well as the electron temperature obtained from the PIC simulation into these terms, the generation of the reversed electric field is found to be mainly caused by the magnetic field term. Due to the electron confinement by the B-field, the electron flux to the electrodes during sheath collapse is limited. Therefore and in order to compensate the positive ion flux to each electrode on time average, an electric field reversal is generated to accelerate electrons towards the electrodes to ensure flux compensation on time average. Once a weak electric field reversal is generated, the electron $\mathbf{E} \times \mathbf{B}$ drift leads to a high electron velocity parallel to the electrodes, $u_{z}$, which causes an increase of the electric field reversal due to an enhancement of the Lorentz force. Increasing the externally applied magnetic field is also found to cause a significant enhancement of the dissociation rate of molecular oxygen. This is explained by the effects of the B-field on the spatio-temporal electron dynamics, for which the magnetically induced electric field reversals play an important role.

Generally, the results of this work quantify the strong effects of externally applied magnetic fields on CCPs operated in $\mathrm{O}_{2}$. They are expected to provide a basis for knowledge based plasma process development and optimization, such as etching on microscopic scales. The electrons accelerated to high energies towards the electrodes by the reversed electric field are able to arrive at the trench bottom in etching processes and neutralize the local positive charge. Thus, the notching effect can be reduced and the trench profile can be improved in such applications [1]. Moreover, our findings are applicable to magnetically enhanced reactive ion etching (MERIE) and RF magnetron sputtering, as they allow to understand the fundamentals of the operation of these plasma sources as a function of the magnetic field. Based on these fundamental insights, concepts to control these plasma sources could be developed and the formation of energy distribution functions of specific process relevant particle species could be optimized. Generally, the results presented in this paper are expected to serve as a basis for additional studies of magnetized RF plasmas in the future. Clearly, a variety of other topics should be addressed. Most importantly, more complex reactive and application relevant gas mixtures as well as more complicated reactor geometries should be investigated, where the reactor sidewall and its interaction with the magneti- 
cally induced radial electron transport might play an important role [68, 69]. Such studies, however, require the application of multi-dimensional Particle-in-Cell simulations.

\section{Acknowledgements}

We thank Trevor Lafleur and Mate Vass for useful discussions on the model of the electric field generation. This work was supported by the National Natural Science Foundation of China (Grant No. 11675036; 11975067), China Scholarship Council (No. 201906060024), the Fundamental Research Funds for the Central Universities (Grant Nos. DUT18TD06, DUT20LAB201), by the German Research Foundation in the frame of the project "Plasmabasierte Prozessführung von reaktiven Sputterprozessen" (No. 417888799), by the National Office for Research, Development and Innovation of Hungary (NKFIH) via grants K-119357, K-132158, FK-128924, and by the J. Bolyai Research Fellowship of the Hungarian Academy of Sciences (AD).

\section{References}

[1] Lieberman M A and Lichtenberg A J 2005 Principles of Plasma Discharges and Materials Processing, 2nd Edition (Wiley)

[2] Makabe T and Petrovic Z L 2014 Plasma Electronics: Applications in Microelectronic Device Fabrication, 2nd Edition (CRC Press)

[3] Chabert P and Braithwaite N 2011 Physics of Radio-Frequency Plasmas (Cambridge: Cambridge University Press)

[4] Lieberman M A, Lichtenberg A J, Kawamura E and Marakhtanov A M 2015 Plasma Sources Science and Technology 24055011

[5] Bogdanova M, Lopaev D, Zyryanov S, Voloshin D and Rakhimova T 2018 Plasma Sources Science and Technology $\mathbf{2 7} 025003$

[6] Olevanov M, Proshina O, Rakhimova T and Voloshin D 2008 Phys. Rev. E 78(2) 026404

[7] Donkó Z, Derzsi A, Vass M, Schulze J, Schuengel E and Hamaguchi S 2018 Plasma Sources Science and Technology $\mathbf{2 7} 104008$ 
[8] Lieberman M A, Lichtenberg A J and Savas S E 1991 IEEE Transactions on Plasma Science 19 189-196 ISSN 1939-9375

[9] Lee S H, You S J, Chang H Y and Lee J K 2007 Journal of Vacuum Science 83 Technôlogy A $25455-463$

[10] You S, Hai T, Park M, Kim D, Kim J, Seong D, Shin Y, Lee S, Park G, Lee J and Chang H 2011 Thin Solid Films 5196981 - 6989 ISSN 0040-6090

[11] Bera K, Rauf S, Kenney J, Dorf L and Collins K 2010 Journal of Applied Physics 107053302

[12] Benyoucef D and Yousfi M 2015 Physics of Plasmas 22013510

[13] Lafleur T 2015 Plasma Sources Science and Technology 25013001

[14] Lafleur T, Delattre P A, Johnson E V and Booth J P 2012 Applied Physics Letters 101124104

[15] Schüngel E, Zhang Q Z, Iwashita S, Schulze J, Hou L J, Wang Y N and Czarnetzki U 2011 Journal of Physics D: Applied Physics 44285205

[16] Lafleur T, Boswell R W and Booth J P 2012 Applied Physics Letters 100194101

[17] Yang S, Innocenti M E, Zhang Y, Yi L and Jiang W 2017 Journal of Vacuum Science 83 Technology A 35061311

[18] Yang S, Zhang Y, Wang H Y, Wang S and Jiang W 2017 Plasma Sources Science and Technology 26065011

[19] Oberberg M, Kallähn J, Awakowicz P and Schulze J 2018 Plasma Sources Science and Technology 27105018

[20] Yang S, Zhang Y, Wang H, Cui J and Jiang W 2017 Plasma Processes and Polymers 14 1700087

[21] Oberberg M, Engel D, Berger B, Wölfel C, Eremin D, Lunze J, Brinkmann R P, Awakowicz P and Schulze J 2019 Plasma Sources Science and Technology 28115021

[22] Czarnetzki U, Mussenbrock T and Brinkmann R P 2006 Physics of Plasmas 13123503

[23] Wen D Q, Kawamura E, Lieberman M A, Lichtenberg A J and Wang Y N 2016 Plasma Sources Sci. Technol. 26015007

[24] Sharma S, Kaganovich ID, Khrabrov A V, Kaw P and Sen A 2018 Physics of Plasmas 25 080704

[25] Proshina O V, Rakhimova T V, Rakhimov A T and Voloshin D G 2010 Plasma Sources Science and Technology 19065013

[26] Belenguer P and Boeuf J P 1990 Phys. Rev. A 41(8) 4447-4459 
[27] Booth J P, Curley G, Marić D and Chabert P 2009 Plasma Sources Sci. Technol. 19015005

[28] Derzsi A, Lafleur T, Booth J P, Korolov I and Donkó Z 2015 Plasma Sources Sci. Technol. 25015004

[29] Küllig C, Wegner T and Meichsner J 2015 Physics of Plasmas 22043515

[30] Katsch H M, Sturm T, Quandt E and Döbele H F 2000 Plasma Sources Science and Technology $9323-330$

[31] Schulze J, Derzsi A, Dittmann K, Hemke T, Meichsner J and Donkó Z 2011 Phys. Rev. Lett. 107(27) 275001

[32] Liu Y X, Schüngel E, Korolov I, Donkó Z, Wang Y N and Schulze J 2016 Phys. Rev. Lett. $\mathbf{1 1 6}(25) 255002$

[33] Gudmundsson J T and Ventéjou B 2015 Journal of Applied Physics 118153302

[34] Gudmundsson J T and Proto A 2019 Plasma Sources Science and Technology 28045012

[35] Gudmundsson J T, Snorrason D I and Hannesdottir H 2018 Plasma Sources Science and Technology 27025009

[36] Derzsi A, Bruneau B, Gibson A R, Johnson E, O'Connell D, Gans T, Booth J P and Donkó Z 2017 Plasma Sources Science and Technology 26034002

[37] Gibson A R and Gans T 2017 Plasma Sources Science and Technology 26115007

[38] Gudmundsson J T and Snorrason D I 2017 Journal of Applied Physics 122193302

[39] You K H, Schulze J, Derzsi A, Donkó Z, Yeom H J, Kim J H, Seong D J and Lee H C 2019 Physics of Plasmas 26013503

[40] Turner M M, Hutchinson D A W, Doyle R A and Hopkins M B 1996 Phys. Rev. Lett. 76(12) $2069-2072$

[41] You S J, Kim S S and Chang H Y 2004 Applied Physics Letters 85 4872-4874

[42] You S J, Chung C,W, Bai K H and Chang H Y 2002 Applied Physics Letters 81 2529-2531

[43] Schulze J, Donkó Z, Heil B G, Luggenhölscher D, Mussenbrock T, Brinkmann R P and Czarnetzki U 2008 Journal of Physics D: Applied Physics 41105214

[44] Sato A H and Lieberman/M A 1990 Journal of Applied Physics 68 6117-6124

[45] Czarnetzki U, Luggenhölscher D and Döbele H F 1999 Plasma Sources Science and Technology $8230-248$

[46] Horváth B, Daksha M, Korolov I, Derzsi A and Schulze J 2017 Plasma Sources Sci. Technol. 26124001 
[47] Campanell M D, Khrabov A V and Kaganovich I D 2012 Phys. Rev. Lettl 108255001

[48] Campanell M D 2013 Phys. Rev. E 88033103

[49] Kushner M J 2003 Journal of Applied Physics 94 1436-1447

[50] Yeom G Y, Thornton J A and Kushner M J 1989 Journal of Applied Physics 65 3825-3832

[51] Schulze J, Donko Z, Derzsi A, Korolov I and Schuengel E 2015 Plasma Sourc. Sci. Technol. 24015019

[52] Vass M, Wilczek S, Lafleur T, Brinkmann R P, Donkó Z and Schulze J 2020 Plasma Sources Science and Technology 29025019

[53] Derzsi A, Korolov I, Schüngel E, Donkó Z and Schulze J 2015 Plasma Sources Sci. Technol. 24034002

[54] Gudmundsson J T, Kawamura E and Lieberman M A 2013 Plasma Sources Sci. Technol. 22 035011

[55] Wang L, Wen D Q, Zhang Q Z, Song Y H, Zhang Y R and Wang Y N 2019 Plasma Sources Sci. Technol. 28055007

[56] Greb A, Gibson A R, Niemi K, O'Connell D and Gans T 2015 Plasma Sources Science and Technology 24044003

[57] Greb A, Niemi K, O'Connell D and Gans T 2013 Appl. Phys. Lett. 103244101

[58] Donkó Z, Derzsi A, Korolov I, Hartmann P, Brandt S, Schulze J, Berger B, Koepke M, Bruneau B, Johnson E, Lafleur T, Booth J P, Gibson A, O'Connell D and Gans T 2018 Plasma Phys. Control. Fusion 60014010

[59] Birdsall C and Langdon A 2004 Plasma Physics via Computer Simulation Series in Plasma Physics and Fluid Dynamics (Taylor \& Francis) ISBN 9780750310253

[60] Boeuf J P 2017 Journal of Applied Physics 121011101

[61] Trieschmann J, Shihab M, Szeremley D, Elgendy A E, Gallian S, Eremin D, Brinkmann R P and Mussenbrock T 2013 Journal of Physics D: Applied Physics 46084016

[62] Zheng B, Wang K, Grotjohn T, Schuelke T and Fan Q H 2019 Plasma Sources Science and Technology 28 09LT03

[63] Stoffels E, Stoffels W W, Vender D, Kando M, Kroesen G M W and de Hoog F J 1995 Phys. Rev. E 51(3) 2425-2435

[64] Küllig C, Dittmann K and Meichsner J 2010 Plasma Sources Science and Technology 19 065011 
[65] Kaga K, Kimura T and Ohe K 2001 Japanese Journal of Applied Physics 40 330-331

[66] Gudmundsson J T, Kouznetsov I G, Patel K K and Lieberman M A 2001 Journal of Physics D: Applied Physics 34 1100-1109

[67] Brinkmann R P 2007 Journal of Applied Physics 102093303

[68] Wen D Q, Kawamura E, Lieberman M A, Lichtenberg A J and Wang Y N 2017 Journal of Physics D: Applied Physics 50495201

[69] Lieberman M A, Lichtenberg A J, Kawamura E and Chabert P 2016 Physics of Plasmas 23 013501 